\title{
Identification, cloning, and functional analysis of the human U6 snRNA-specific terminal uridylyl transferase
}

\author{
RALF TRIPPE, ${ }^{1}$ ELENA GUSCHINA, ${ }^{1}$ MARKUS HOSSBACH, ${ }^{2}$ HENNING URLAUB, ${ }^{3}$ \\ REINHARD LÜHRMANN, ${ }^{2}$ and BERND-JOACHIM BENECKE ${ }^{1}$ \\ ${ }^{1}$ Lehrstuhl für Biochemie I, Ruhr-Universität, Bochum, 44780 Bochum, Germany \\ ${ }^{2}$ Abteilung für Zelluläre Biochemie, Max-Planck-Institut für Biophysikalische Chemie, 37077 Göttingen, Germany \\ ${ }^{3}$ Bioanalytische Massenspectrometrie, Max-Planck-Institut für Biophysikalische Chemie, 37077 Göttingen, Germany
}

\begin{abstract}
Mammalian cells contain a highly specific terminal uridylyl transferase (TUTase) that exclusively accepts U6 snRNA as substrate. This enzyme, termed U6-TUTase, was purified from HeLa cell extracts and analyzed by microsequencing. All sequenced peptides matched a unique human cDNA coding for a previously unknown protein. Domain structure analysis revealed that the U6-TUTase also belongs to the well-characterized poly(A) polymerase protein superfamily. However, by amino acid sequence as well as RNA-binding motifs, human U6-TUTase is highly divergent from both the poly(A) polymerases and from the TUTases identified within the editing complexes of trypanosomes. After cloning, the recombinant U6-TUTase was expressed in HeLa cells. Analysis of its catalytical activity confirmed the identity of the cloned protein as U6-TUTase, exhibiting the same exclusive substrate specificity for U6 snRNA as the endogenous enzyme. That unique selectivity even excluded as substrate U6atac RNA, the functional homolog of the minor spliceosome. Finally, RNAi knockdown experiments revealed that U6-TUTase is essential for cell proliferation. Surprisingly, large amounts of the recombinant enzyme were found to accumulate within nucleoli.
\end{abstract}

Keywords: uridylytransferase; TUTase; RNA modification; U6 snRNA; U6atac RNA

\section{INTRODUCTION}

Most eukaryotic RNA species are synthesized as precursor molecules and subsequently modified in a variety of posttranscriptional processing events. In that respect, one of the most intensively studied processes is the splicing of nuclear pre-mRNA. The removal of introns from primary transcripts is catalyzed by the spliceosome. The composition and structure of this highly conserved large RNP complex now is well established (Hartmuth et al. 2002; Jurica et al. 2002). Among the five small nuclear RNA species involved (U1, U2, U4, U5, and U6 snRNA), U6 snRNA is believed to play a central role in the catalytic steps of pre-mRNA splicing. This notion is supported by its participation in multiple RNA-RNA interactions (Staley and Guthrie 1998; Brow 2002) as well as by the finding that U6 snRNA is the most highly conserved spliceosomal RNA (Brow and Guthrie 1988). U6 snRNA is also unique in that nucleotides

Reprint requests to: Bernd-Joachim Benecke, Lehrstuhl für Biochemie I, NC6, Ruhr-Universität Bochum, D-44780 Bochum, Germany; e-mail: bernd.benecke@rub.de; fax: 49-234-321 4034.

Article published online ahead of print. Article and publication date are at http://www.rnajournal.org/cgi/doi/10.1261/rna.87706. are both added to and removed from its $3^{\prime}$-end (Rinke and Steitz 1985; Booth and Pugh 1997). Furthermore, the majority of cellular U6 snRNA molecules terminate with an unusual 2'-3'-cyclic UMP phosphate (Lund and Dahlberg 1992). It was speculated that some of those modifications may be functionally related to U6 snRNA activity within the spliceosome (Tazi et al. 1993). Though being synthesized by RNA polymerase III (Kunkel et al. 1986; Reddy et al. 1987; Dahlberg and Lund 1988), important steps of U6 snRNA maturation have been shown to take place within the nucleolus (Tycowski et al. 1998; Ganot et al. 1999).

In addition to adenylating enzymes such as poly(A) polymerases (PAP), eukaryotic cells also possess transferases known to uridylate RNA. Three distinct groups of terminal uridylyl transferases (TUTases) can be distinguished: The first group is associated with the editing complexes found in mitochondria of trypanosomes. Directed by guide RNAs (gRNAs), these very intensively studied enzymes catalyze the U-insertion/deletion reaction that specifically modifies the nucleotide sequence of mRNA transcripts (Aphasizhev et al. 2002, 2003; Ernst et al. 2003; Panigrahi et al. 2003). A second, yet less well-characterized enzyme is involved in poliovirus replication. This cellular host factor provides 
the oligo(U) primer required for the initiation of RNA synthesis by the virus-encoded RNA-dependent RNA polymerase (Andrews and Baltimore 1986). On the average, this host enzyme adds five uridine residues, with no clear preference for virion RNA. A potentially related cellular TUTase described in plants (Zabel et al. 1981) revealed no pronounced substrate specificity, as well. However, the transferase reaction was strictly limited to the addition of a single UMP residue. Regarding that aspect, the plant enzyme seems to resemble the signal recognition particle (srp) RNA adenylating enzyme that was cloned recently (Perumal et al. 2001). The third type of eukaryotic TUTases was detected in in vitro transcription experiments with HeLa cell nuclear extracts. In a template-independent reaction, that enzyme catalyzed the uridylation of U6 snRNA. The exceptionally high specificity of this reaction is indicated by two criteria: only U6 snRNA was accepted as substrate (Trippe et al. 1998), and the transferase reaction was strictly controlled in elongation (Trippe et al. 2003). As a result of this post-transcriptional modification, the four 3 '-terminal UMP residues are restored, as found in newly transcribed U6 snRNA. This finding of an "own" modifying enzyme also is in support of the above-described exceptional status of U6 snRNA.

In this paper, we describe the identification of the human U6 snRNA-specific TUTase gene and its transient expression in HeLa cells. The recombinant protein was clearly identified by its terminal uridylyl transferase activity, retaining its high selectivity with U6 snRNA being the only substrate accepted among total cellular RNA. Our results indicate that this U6 snRNA modifying enzyme may accumulate within nucleoli. Furthermore, RNA interference experiments provide evidence that the U6-TUTase constitutes an essential cellular protein.

\section{RESULTS}

\section{Purification of U6-TUTase}

Former experiments had shown that HeLa cells contain two distinct TUTase activities which can be separated by ion exchange chromatography (Trippe et al. 1998). In our attempts to clone the U6 snRNA-specific enzyme, cellular extracts were purified according to the procedure outlined in detail previously (Trippe et al. 2003). Briefly, S100 extracts were fractionated by Q-Sepharose chromatography, and the step-eluted QS3 fraction (200-400 mM KCl) was further purified with hydroxyapatite. Subsequently, individual fractions $(30 \mu \mathrm{L})$ were assayed for TUTase activity, using $50 \mathrm{ng}$ of purified U6 snRNA alone or $1 \mu \mathrm{g}$ of total cellular RNA as substrate (Fig. 1A). TUTase assays were performed in buffer containing $60 \mathrm{mM} \mathrm{KCl}, 12 \mathrm{mM}$ HEPES/KOH (pH 7.9), 5 mM $\mathrm{MgCl}_{2}, 2 \mathrm{mM}$ dithiothreitol, $0.1 \mathrm{mM}$ EDTA, $12 \%(\mathrm{v} / \mathrm{v})$ glycerol, and $5 \mu \mathrm{Ci}\left[\alpha-{ }^{32} \mathrm{P}\right] \mathrm{UTP}$, in a total volume of $50 \mu \mathrm{L}$. When analyzed with U6-3 RNA
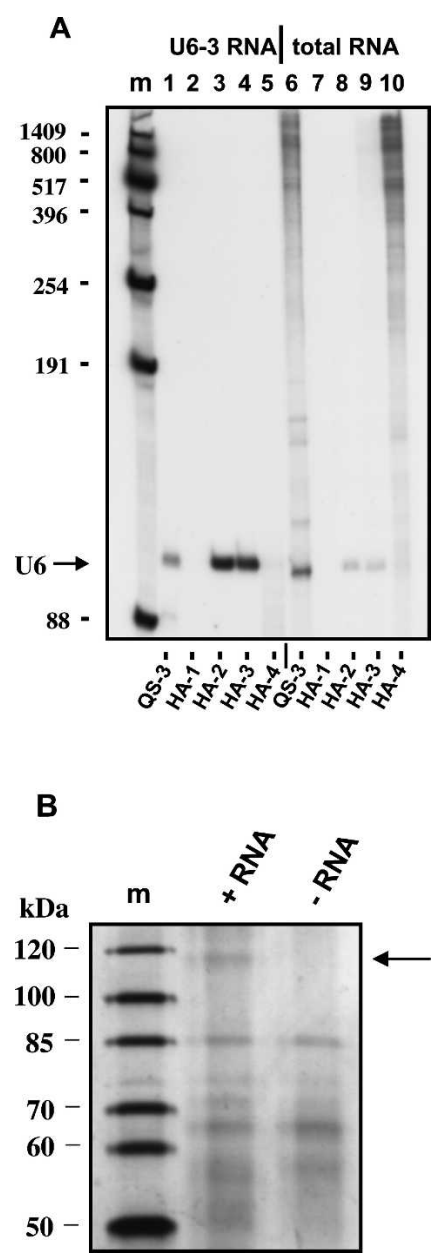

FIGURE 1. Isolation of HeLa cell U6-TUTase from subcellular fractions. (A) Analysis of TUTase activity with either in vitro synthesized U6-3 RNA or total HeLa cell RNA as substrate. Fractions were step-eluted from Q-Sepharose (QS-3, 200-400 $\mathrm{mM} \mathrm{KCl)} \mathrm{or}$ hydroxyapatite columns (HA) with HA-1 corresponding to $15 \mathrm{mM}$, HA-2 to $75 \mathrm{mM}, \mathrm{HA}-3$ to $150 \mathrm{mM}$, and HA-4 to $500 \mathrm{mM}$ potassium phosphate, respectively. After modification, phenol-extracted RNAs were analyzed in $6 \%$ polyacrylamide gels containing $6 \mathrm{M}$ urea. The position of U6 snRNA is indicated by an arrow. The length of labeled DNA marker fragments $(m)$ is indicated on the left. $(B)$ Affinity purification of U6-TUTase (HA-2 fraction) with oligo $\left(\mathrm{A}_{20}\right) / \mathrm{U} 6-3$ RNA immobilized on oligo(dT)-cellulose (+RNA column). Proteins eluted in parallel from oligo(dT)-cellulose alone (-RNA column) were analyzed as a control. Molecular mass markers are indicated on the left; the position of the U6-TUTase is marked by an arrow.

as substrate, the starting QS-3 fraction (Fig. 1A, lane 1) was clearly active in U6 snRNA modification. However, TUTase assays performed with that fraction and total cellular RNA (lane 6) resulted in a complex spectrum of labeled RNA products. In addition to U6 snRNA, a variety of larger RNA species was labeled, indicating the activity of an unspecific uridylating enzyme. It should be noted that the slightly different migration observed with in vitro synthesized U6-3 RNA versus cellular U6 RNA is due to two additional G-residues required for in vitro transcription by $\mathrm{T} 7$ 
RNA polymerase (see Materials and Methods). Different results were obtained upon chromatography with hydroxyapatite. That purification step clearly separated two cellular TUTase enzymes. The analysis of step-eluted fractions revealed that virtually all TUTase bound to the column, with no activity detectable in the flow-through fraction HA-1 (Fig. 1A, lanes 2,7$)$. In contrast, TUTase activity was detected in the step-eluted HA-2 (75 mM potassium phosphate), HA-3 (150 mM), and HA-4 (500 mM) fractions. The analysis of substrate specificity with U6-3 RNA alone (Fig. 1A, lanes 2-5) or total cellular RNA (lanes 7-10) confirmed that the two TUTase activities were associated with different fractions. The HA-2 fraction (lanes 3,8) exclusively contained U6-TUTase. In contrast, the HA-4 fraction showed very little, if any, modification of U6-3 RNA (lane 5). Rather, when analyzed with total RNA as substrate, a complex spectrum of larger labeled RNA molecules was observed with the HA-4 fraction (lane 10). This result is reminiscent of the former described unspecific cellular TUTase (Andrews and Baltimore 1986). Comparing lanes 4 and 9 of Figure 1A, the HA-3 fraction also contained significant amounts of the U6 snRNA-specific TUTase, however, slightly contaminated with the unspecific enzyme (lane 9).

Subsequently, the HA-2 fraction was further purified by affinity chromatography, with U6 snRNA as bait, coupled to oligo(dT)-cellulose. Proteins eluted from that U6 snRNA affinity-column are shown in Figure 1B. Compared to proteins recovered from oligo(dT)-cellulose alone (-RNA), only one prominent polypeptide was specifically obtained from the U6 snRNA column (+RNA). That band (arrow), migrating with an apparent molecular mass of $115 \mathrm{kDa}$, was virtually absent in the corresponding elution fraction of the minus-RNA column.

\section{Identification of the U6-TUTase protein}

A protein band as shown in Figure 1B (arrow) was cut from the gel, eluted, and subjected to trypsin digestion. Several peptides were analyzed by microsequencing. A search against the available protein and expressed sequence tag databanks revealed that almost all peptides matched a unique human cDNA corresponding to the hypothetical protein FLJ22347 of unknown function. The sequence of the corresponding cDNA, now identified as the human U6-TUTase, had been submitted to GenBank with the accession number AK026000. That cDNA is 2747 bp in length, containing a 2622-nt open reading frame coding for 874 amino acids with a deduced molecular mass of $93.8 \mathrm{kDa}$ for the resulting protein. The coding region is preceded by $59 \mathrm{nt}$ of $5^{\prime}$-untranslated leader sequence with a purine at position -3 of the initiator AUG codon, constituting an adequate Kozak consensus sequence. An authentic polyadenylation signal, AAUAAA, is found 20 bp downstream of the stop codon. The gene coding for this protein has a total length of $\sim 17 \mathrm{~kb}$ and is located on Chromosome 11. The expressed sequence is divided into nine exons, preferentially located within the $3^{\prime}$-half of the transcription unit.

According to the results obtained by NCBI-BLAST search, the U6-TUTase contains two functional domains (Fig. 2). An RNA-binding domain, identified near the amino terminus, consists of a C2-H2 zinc finger motif (amino acids 14-47), closely followed by an RNA recognition motif (RRM; amino acids 57-11) of the RNP-1 type. The second functional domain is located within the center of the protein, comprising a TRF4 element (amino acids 357-560) with significant homology with DNA polymerase $\sigma$. That motif overlaps with a PAP-associated PAP/25A domain (amino acids 490-548), known from poly(A) polymerases. The latter two motifs identify the U6-TUTase as a new member of nucleotidyl transferases, with TRF4 and PAP/25A very likely representing the catalytic domain of the enzyme. The domain structure of the human U6-TUTase depicts a clear relation of this enzyme to the $\operatorname{poly}(\mathrm{A})$ polymerase superfamily, which is also supported by the schematic presentation in Figure 2. Here, the identified domains of the U6-TUTase are compared with human poly(A) polymerase $\alpha$ (Zhelkovsky et al. 1995; Martin and Keller 1996) and the terminal uridylyl transferase associated with the editing complex of Trypanosoma brucei (Aphasizhev et al.
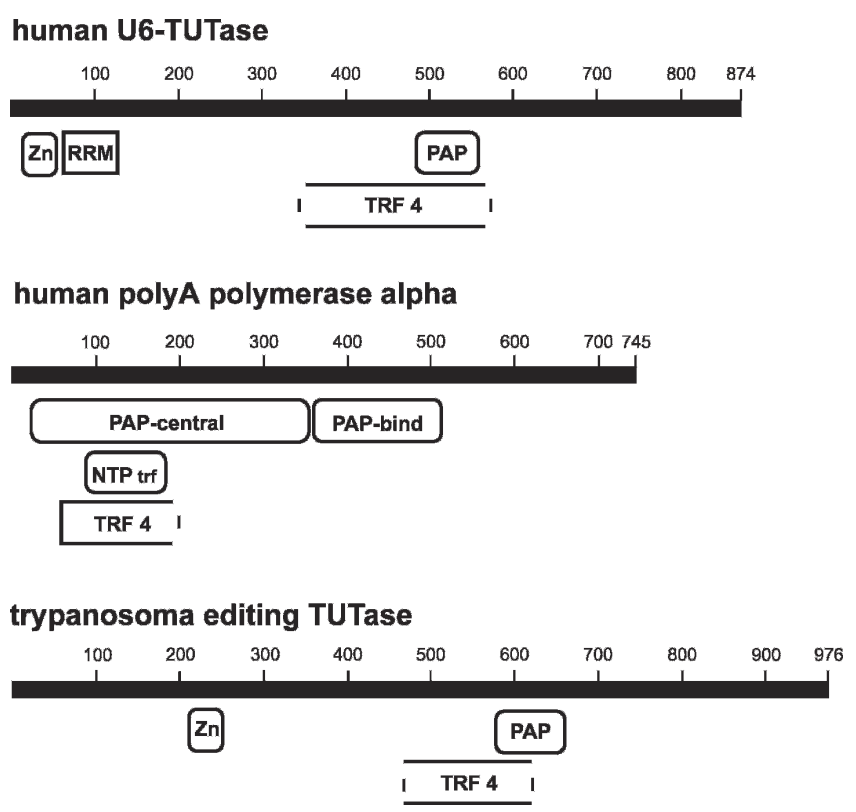

FIGURE 2. Schematic domain structures of selected nucleotidyl transferases. Identified domains were redrawn from the results obtained by the NCBI "conserved domain database." Abbreviations refer to Zn, zinc finger motif; RRM, RNA recognition motif; PAP, poly(A) polymerase-associated domain; TRF4, DNA polymerase sigma domain; PAP-central, poly(A) polymerase central domain; PAP-bind, poly(A) polymerase predicted RNA-binding domain; NTP trf, nucleotidyltransferase domain. Trypanosoma editing TUTase refers to the RET 1 enzyme of Trypanosoma brucei (Aphasizhev et al. 2002). 
2002). All three proteins share the PAP-associated and TRF4 domains. The relative positions of these elements are very similar among the two uridylating enzymes, possibly indicating a common ancestor. However, differences are observed with respect to the RNA-binding domains. The RNA-binding region of the poly (A) polymerase (PAP-bind) is located at the carboxy-terminal side, adjacent to the catalytic domain. In contrast, the putative RNA-binding domain of the human U6-TUTase is located at the very amino-terminal side of the protein, whereas no such RNAbinding motif was identified by domain search within the trypanosomal editing TUTase. Among each other, the three proteins did not reveal any extended amino acid sequence homology (data not shown).

\section{Expression of the recombinant U6-TUTase in mammalian cells}

Following its identification, the cDNA of the human U6TUTase was cloned into an eukaryotic expression vector and transiently transfected into HeLa cells. For these experiments, the gene construct was supplemented with a His-tag and a myc-tag, both located at the carboxyl terminus of the protein. The Western blot analysis with anti-myc antibody of extracts isolated from transiently transfected HeLa cells is shown in Figure 3A. In addition to some weaker signals which were also observed with nontransfected cells (lane 1), a strong reaction of the monoclonal antibody was obtained with extracts from transfected cells (lanes 2,3). The protein identified corresponded in size to the purified human U6-TUTase (cf. Fig. 1B). The extracts analyzed in lanes 2 and 3 of Figure $3 \mathrm{~A}$ resulted from two independent experiments, with lane 2 representing an analytical experiment, while lane 3 shows the result of a large-scale transfection experiment. Larger amounts of the recombinant protein were required for a functional analysis of the cloned enzyme. For this, the proteins of the S100 extract were applied to a nickel-NTA affinity-column under native conditions and step-eluted with imidazole. As evident from the Western blot analysis in Figure 3B, the vast majority of the recombinant protein present within the load fraction ( $l$ ) was bound to the column, with only small amounts detectable in the flow-through fraction $(\mathrm{ft})$ and almost none in the wash fraction $(w)$. Step-eluted fractions were obtained from the column with increasing concentrations of imidazole. The recombinant protein eluted in a broad peak with the majority found between $30 \mathrm{mM}\left(\mathrm{e}_{1}\right)$ and $75 \mathrm{mM}\left(\mathrm{e}_{3}\right)$ of imidazole. Only residual amounts of U6-TUTase were eluted at higher imidazole concentrations $\left(\mathrm{e}_{4}=100 \mathrm{mM}\right.$ and $\left.\mathrm{e}_{5}=200 \mathrm{mM}\right)$.

Parallel to its expression in HeLa cells, human U6TUTase was expressed in Escherichia coli. In order to raise polyclonal antibodies, recombinant protein was purified by affinity-chromatography and preparative gel electrophoresis and used for immunization of rabbits (data not shown).
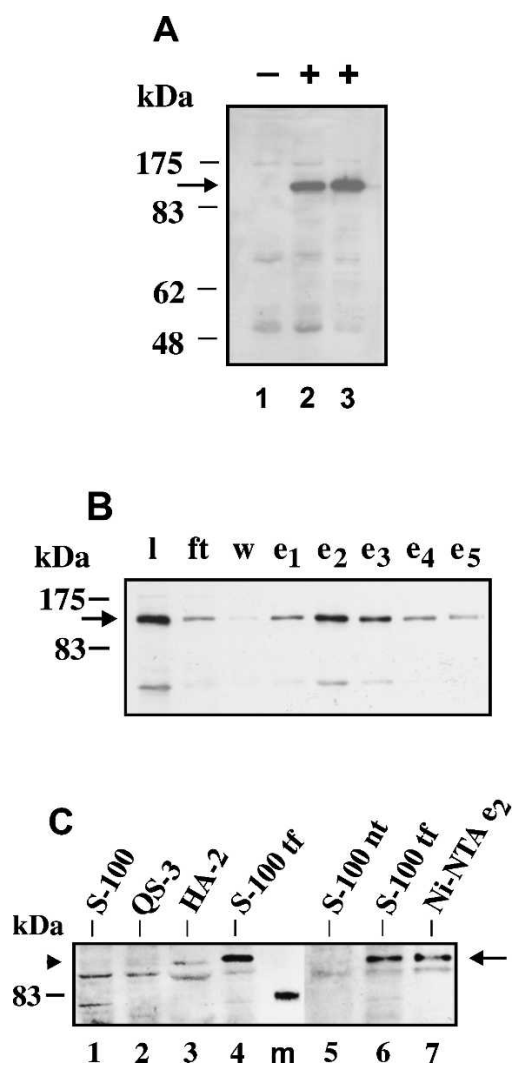

FIGURE 3. Western blot analysis of U6-TUTase expressed in cultured mammalian cells. The cDNA coding for U6-TUTase was supplemented with myc- and His-tag sequences and cloned into the T-Rex expression vector. (A) After transfection of HeLa cells, S100 extract proteins were separated by gel electrophoresis, transferred to nitrocellulose membranes, and analyzed with a monoclonal c-myc antibody. (Lane 1) Untransfected control cells (-); (lane 2) trans-

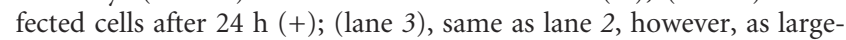
scale preparation (20 dishes) for subsequent enzyme purification. Expressed U6-TUTase is indicated by an arrow. Molecular masses are indicated on the left. (B) Proteins of the large-scale preparation in part A (lane 3) were subjected to affinity chromatography in a Ni-NTA column. Proteins associated with the load $(l)$, flow-through $(\mathrm{ft})$, wash $(w)$, and elution-fractions $\left(\mathrm{e}_{1-5}\right)$ were separated and analyzed with c-myc antibodies as above. Elution was with $30 \mathrm{mM}\left(\mathrm{e}_{1}\right), 50 \mathrm{mM}\left(\mathrm{e}_{2}\right)$, $75 \mathrm{mM}\left(\mathrm{e}_{3}\right), 100 \mathrm{mM}\left(\mathrm{e}_{4}\right)$, and $200 \mathrm{mM}\left(\mathrm{e}_{5}\right)$ imidazole, respectively. The load fraction was adjusted to $5 \mathrm{mM}$ imidazole and washed with $10 \mathrm{mM}$. Again, molecular mass markers and the position of the U6-TUTase (arrow) are indicated on the left. (C) Western blot analysis of subcellular fractions with rabbit polyclonal antibodies raised against U6-TUTase. After blotting, fractions representing different steps of the purification procedure described before were analyzed with U6-TUTase antibodies. Fractions in lanes 1, 2, 3, and 5 were from non-transfected (nt) HeLa cells, whereas fractions in lanes 4, 6, and 7 were obtained from HeLa cells transiently transfected (tf) with the U6-TUTase expression plasmid. The slightly larger size of the recombinant TUTase (arrow; cf. lanes 3,4) is due to the presence of the two (myc and His) tags. The positions of the endogenous U6TUTase (arrowhead) and a marker protein are indicated on the left.

Subsequently, affinity-purified antibodies were assayed in Western blots with HeLa cell subfractions. As shown in lanes 1-3 of Figure 3C, only weak signals were detectable for the endogenous U6-TUTase (arrowhead) in S100, QS-3, 
and HA-2 fractions of non-transfected (nt) cells, respectively. In contrast, transfection of HeLa cells (tf) with the U6-TUTase expression plasmid resulted in a several-fold increase in detectable U6-TUTase within S100 extracts (Fig. $3 \mathrm{C}$, lane 4). It should be noted that the slightly slower migration of the recombinant U6-TUTase (cf. lanes 3,4) is due to the presence of the two (myc- and His-) tags, needed for purification and detection. Furthermore, the comparison of lanes 1, 5 with 4, 6 of Figure 3C indicates that the endogenous U6-TUTase basically represents only a fairly low-abundant protein in wild-type HeLa cells. This assumption is based on the observation that expression of the recombinant protein, even under transient transfection conditions, resulted in a several-fold increase in enzyme concentration. As observed before with the monoclonal myc-antibody (see Fig. 3B), the affinity-purified U6-TUTase $\left(e_{2}\right.$ fraction, lane 7$)$ was identified also with this polyclonal rabbit antibody.

Subsequently, fractions affinity-purified from transfected HeLa cells were processed and analyzed for TUTase activity, using in vitro synthesized U6-3 snRNA as substrate. The results in Figure 4A show that the recombinant enzyme definitely exhibited uridylating activity in vitro. The U6TUTase activity observed with individual fractions paralleled almost exactly the distribution of the myc-tagged protein identified in the Western blot before (Fig. 3B). With their apparently low uridylating activities, only the fractions eluted at higher imidazole concentrations $\left(\mathrm{e}_{4}\right.$ and $e_{5}$ ) slightly differed from the Western blot results. Although those uridylation signals were hardly seen on the original film, the Western blots before had confirmed that both fractions contain detectable amounts of U6-TUTase. That
A

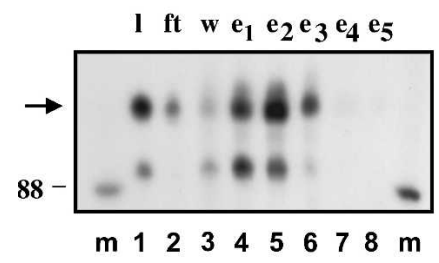

B

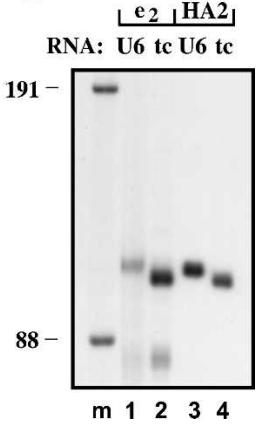

FIGURE 4. Functional analysis of the human U6-TUTase expressed in HeLa cells. (A) The affinity-purified protein fractions (Fig. 3B) were dialyzed to remove imidazole and subsequently analyzed for TUTase activity with in vitro synthesized U6-3 RNA as substrate. A 88-nt marker band and the position of modified U6 snRNA (arrow) are indicated on the left. (B) Comparison of substrate specificity of recombinant and cellular U6-TUTases. Affinity-purified TUTase ( $\mathrm{e}_{2}$-fraction) and the endogenous enzyme (HA2-fraction) were assayed in parallel with U6-3 RNA (U6, lanes 1,3) or total cellular RNA (tc, lanes 2,4) as substrate. The positions of DNA marker bands are indicated on the left. discrepancy was reproducibly observed, and it is quite conceivable that the transient exposure to higher amounts of imidazole mediates a negative effect on proper folding of the protein, resulting in reduced enzyme activities even after removal of the reagent. Alternatively, an inhibitor might be eluted at higher imidazole concentrations. In the TUTase assay, the recombinant protein generated an additional labeled RNA product of $\sim 90 \mathrm{nt}$ in length. That reproducible shorter band was not observed with the cellular enzyme and probably represents a degradation product due to imidazole treatment.

With the unambiguous identification of the cloned protein as a uridylating enzyme, a second important feature remained to be confirmed. The most distinctive property of the human U6-TUTase consists in its unique substrate specificity: U6 snRNA alone is accepted for uridylation (Trippe et al. 1998, 2003). Therefore, it was crucial to see whether or not the recombinant enzyme had retained that substrate specificity. For this reason, affinity-purified U6TUTase ( $e_{2}$ fraction) was assayed either with in vitro synthesized U6-3 RNA (U6) or with phenol-extracted total cellular RNA (tc) as substrate. For a comparison, that experiment was performed in parallel with the cellular U6TUTase (HA-2 fraction). As is evident from a comparison of lanes 1, 2 with lanes 3, 4 of Figure 4B, both TUTase fractions showed exactly the same substrate specificity. Like the cellular enzyme (lane 3), the cloned TUTase revealed a clear uridylation signal with purified U6-3 snRNA (lane 1). Furthermore, among total cellular RNA (tc, lanes 2,4) both enzyme fractions exclusively selected U6 snRNA for modification (again, the apparent difference in length between U6-3 RNA and cellular U6 snRNA is due to the presence of two additional 5'-GMP residues required for T7 transcription). In particular within the size range between U6 snRNA and the 191-nt marker band, no further labeled RNA product was obtained, as observed, for example, with the unspecific uridylating enzyme (see Fig. 1A, lane 6). The only additional labeled bands are those smaller degradation products, reproducibly obtained with the recombinant enzyme (see above). The weaker U6 snRNA signal seen in lane 1 of Figure $4 \mathrm{~B}$ was due to some incomplete dissolution of the extracted RNA, since here significant amounts of label remained in the gel slot (data not shown). Together, these results confirmed that upon expression in HeLa cells, the cloned enzyme revealed the same major characteristics as observed with the cellular U6-TUTase.

\section{U6atac RNA is not a substrate of the human U6-TUTase}

With respect to the above-mentioned pronounced substrate specificity of the U6-TUTase, an intriguing question arose during the course of this investigation. That notion aimed at the U6atac RNA of the minor spliceosome (for review, see Patel and Steitz 2003). Since U6 snRNA and 
U6atac RNA are functional homologs, it was interesting to see whether or not U6-TUTase may also be involved in U6atac RNA 3'-end modification. For this reason, cellular U6-TUTase was assayed with both U6-3 RNA and U6atac RNA as substrate, respectively. As is evident from the comparison of lanes 2 and 4 of Figure 5, absolutely no uridylation of U6atac RNA by U6-TUTase was detectable, whereas U6-3 RNA again was effectively modified by the addition of one labeled UMP-residue (lane 4). For both reactions, the unmodified substrate RNAs, labeled during in vitro transcription by T7 RNA polymerase, were included as standards (see lanes 1,3). It should be noted that in this experiment the unlabeled U6atac RNA too carried three U-nucleotides at its $3^{\prime}$-end.

\section{U6-TUTase constitutes an essential cellular protein}

The most intriguing question arising from the finding of a distinct U6-TUTase aims at the biological significance of that modifying enzyme. To address that point, RNA interference (RNAi) experiments were performed. HeLa cells were transfected with double-stranded siRNA directed against U6-TUTase mRNA. In a first step, cell proliferation was determined up to $72 \mathrm{~h}$ post-transfection. As is shown in Figure 6A, HeLa cells transfected with $\Delta U 6$-TUTase revealed reduced proliferation rates already at $48 \mathrm{~h}$. At $72 \mathrm{~h}$, viability was down to $25 \%$ of control cells, transfected with siRNA directed against firefly luciferase mRNA (GL2). As a positive control, HeLa cells were transfected in parallel with $\Delta$ hPrp8 siRNA aiming at the known essential protein factor Prp8 of the human spliceosome (for review, see Grainger and Beggs 2005). The viability rate observed after knockdown of this spliceosomal factor almost exactly

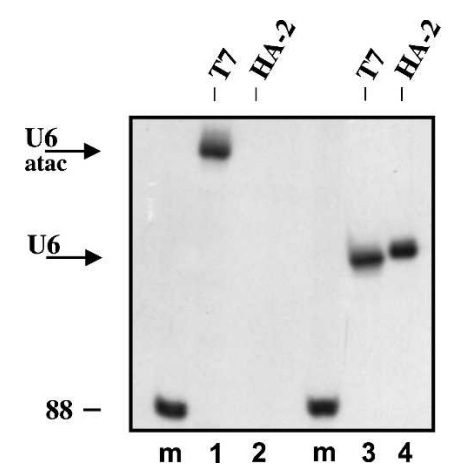

FIGURE 5. Analysis of the cellular U6-TUTase with different U6 RNA substrates. The partially purified endogenous U6-TUTase (HA-2 fraction) was assayed in parallel with U6-3 RNA (lane 4) or U6atac RNA (lane 2), also containing three terminal UMP-residues at its $3^{\prime}$-end. For a comparison, lanes 1 and 3 show the in vitro synthesized U6atac and U6-3 RNAs, respectively, labeled during T7 RNA polymerase transcription (T7). The same, yet unlabeled RNAs were used as substrate for the TUTase reaction. The positions of the nonmodified labeled RNAs (arrows) and the marker band are indicated on the left.
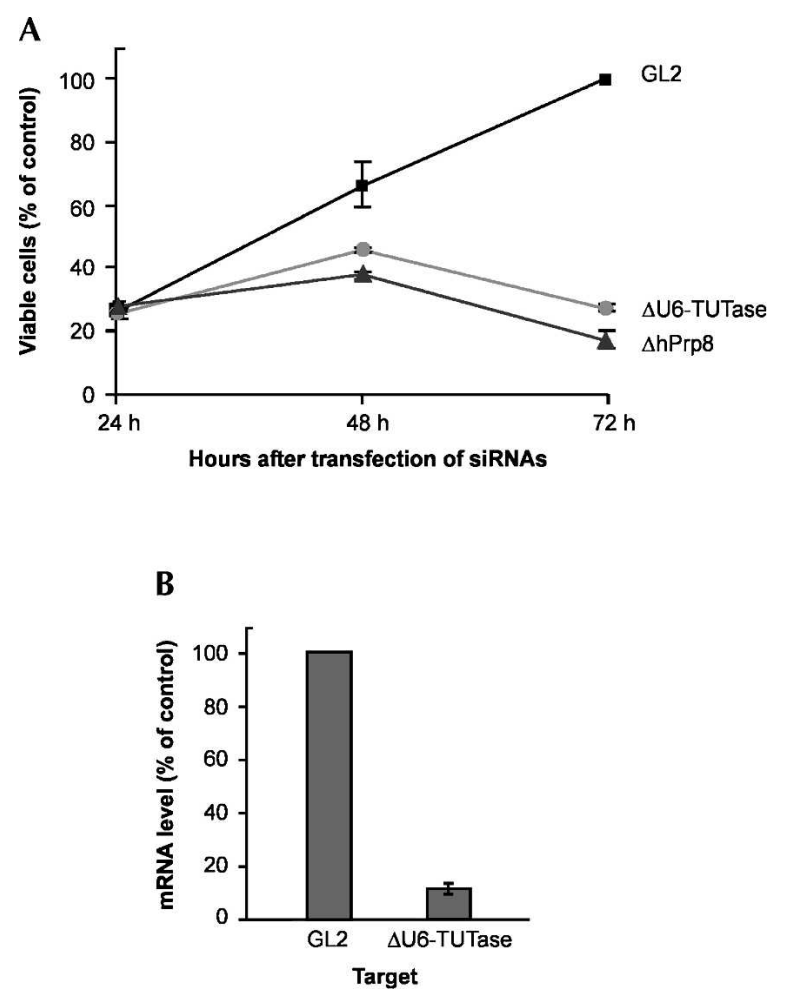

FIGURE 6. RNA interference of U6-TUTase expression. (A) RNAi knockdown reveals essential cellular function of the enzyme. Cell proliferation was determined at $24 \mathrm{~h}, 48 \mathrm{~h}$, and $72 \mathrm{~h}$ after transfection of double-stranded siRNA directed against U6-TUTase mRNA. The average of twofold determinations from duplicate experiments is depicted as the percentage of viable cells of the control knockdown (siRNA against GL2 luciferase) at $72 \mathrm{~h}$ after transfection. As positive control, knockdown was performed with siRNA directed against the hPrp8 protein, known to be essential for the catalytic steps I and II in pre-mRNA splicing. (B) Analysis by real-time RT-PCR of U6-TUTase mRNA levels in response to RNAi knockdown. HeLa SS6 cells grown in six-well cell culture dishes were transfected with either GL2 (control) siRNA or siRNA directed against U6-TUTase at a concentration of $110 \mathrm{nM}$. After $48 \mathrm{~h}$, total RNA was isolated and treated with DNase I. Then, $12.5 \mathrm{ng}$ of total RNA was subjected to one-step RT-PCR (QuantiTec SYBR Green RT-PCR kit, QIAGEN). The graph shows the U6-TUTase mRNA levels as calculated from the real-time PCR data. Control cells (transfected with GL2 siRNA, 100\%, left) are compared with cells transfected with siRNA directed against U6-TUTase mRNA (right). Error bars indicate the standard deviation for three independent experiments.

paralleled that observed with the $\Delta$ U6-TUTase siRNA. These data indicate that the U6-TUTase constitutes an essential cellular protein. To verify directly the breakdown of U6-TUTase mRNA by siRNA, real-time RT-PCR was performed. Figure $6 \mathrm{~B}$ shows the U6-TUTase mRNA levels detected $48 \mathrm{~h}$ after transfection of HeLa cells with either control siRNA (GL2) or with U6-TUTase-specific sequences ( $\Delta$ U6-TUTase). As before, only the specific siRNA revealed a strong effect on U6-TUTase mRNA levels, resulting in $>80 \%$ reduction as compared to control cells. This result confirmed that the selected siRNA duplex (see Materials 
and Methods) effectively mediates U6TUTase mRNA degradation.

\section{Predominant nucleolar localization of expressed U6-TUTase}

Finally, experiments were performed to study the intracellular localization of U6-TUTase by indirect immunofluorescence. Therefore, samples of HeLa cells transfected with the U6-TUTase expression plasmid were processed and incubated with monoclonal mouse antibodies directed against the c-myctag of the recombinant protein. Visualization of the protein with an Alexa488-labeled secondary antibody revealed an unexpected result. A strong accumulation of green fluorescence was detectable in the nucleolar region. To confirm the observed predominant nucleolar localization of the recombinant U6-TUTase, processed cells were additionally analyzed with primary antibodies directed against the known U3 snoRNP protein NOP58 (Watkins et al. 2000). Here, a Texas Red conjugated antirabbit IgG as secondary antibody was used for detection of that nucleolar marker protein. The upper line of Figure 7 shows the result of such a double-stain experiment. Recombinant U6-TUTase was detected throughout the nucleus, yet with strong accumulation within the nucleoli (Fig. 7A). As expected, the nucleolar marker protein NOP58 was exclusively confined to the nucleolus (Fig. 7B). The colocalization of both proteins within the nucleolus is clearly visible by the bright orange dots in the merged images in Figure 7, C-E.

In a second double-stain experiment, the localization of U6-TUTase was visualized in comparison to p110. Recently, that protein was identified as a U4/U6 snRNP recycling factor which transiently associates with U6 snRNA (Damianov et al. 2004). Furthermore, p110 was found distributed throughout the nucleoplasm, but was highly enriched in Cajal bodies (Stanek et al. 2003). The immunofluorescent labeling experiment shown in the lower line of Figure 7 confirmed that localization of p110. As seen in Figure 7G, p110 revealed an extended distribution throughout the nucleoplasm, yet with a distinct accumulation within Cajal bodies (bright red dots). Furthermore, that protein was completely absent from nucleoli. The merged images of this double-stain experiment (Fig. $7 \mathrm{H}-\mathrm{J}$ ) revealed a certain colocalization of p110 and U6-TUTase within the nucleoplasm (orange staining). In contrast, the predominant localization of both proteins, however, clearly identified two separate subnuclear structures: U6-TUTase in nucleoli (green) versus p110 in Cajal bodies (red). Together, these findings support the notion that the complex pathway of U6 snRNA recycling involves shuttling between several distinct subnuclear compartments.

\section{DISCUSSION}

This study presents data on the identification, cloning, and functional analysis of the U6 snRNA-specific terminal uridylyl transferase (Trippe et al. 1998). That human U6TUTase is one of only two RNA-uridylating enzymes of vertebrates that have been characterized in detail so far (Andrews and Baltimore 1986; Trippe et al. 2003) and up to now the first one to be cloned. Based on its catalytical activity, the enzyme clearly belongs to the widespread and still growing superfamily of nucleotidyl transferases which are involved in a variety of metabolic pathways (Aravind and Koonin 1999). A characteristic feature of that superfamily is the TRF 4 domain of DNA polymerase $\sigma$. A subgroup of those nucleotidyl transferases is involved in posttranscriptional modification of a variety of RNA species. Among others, that subgroup includes CCA-adding enzymes, TUTases, and poly(A) polymerases. The latter enzymes are 
believed to represent the typical domain structure of RNAmodifying nucleotidyl transferases. In addition to the TRF4 domain, these enzymes contain the catalytic PAP domain found in all three classes of poly(A) polymerases.

Therefore, the human U6-TUTase was compared with other enzymes known to catalyze similar RNA-modifying nucleotidyl transferase reactions. For this comparison, two well-characterized enzymes were of particular interest, the trypanosomal RNA editing TUTase (RET 1) and the human poly(A) polymerase (PAP). Aside from the aforementioned TRF 4 and PAP domains, all three enzymes showed only very limited structural similarity. The major difference seems to be related to the location of the RNAbinding domain. In poly(A) polymerases, the RNA-binding domain (PAP-RNA-bind) is located at the C-terminal side, immediately adjacent to the PAP core section. In contrast, a putative RNA-binding domain of the human U6-TUTase, an RNA recognition motif (RRM) of the RNP1-type, was found near the N-terminus of the protein. An NCBI-BLAST domain search of the Trypanosoma brucei TUTase did not point to any RNA-binding motif. However, a recent study (Aphasizheva et al. 2004) mapped an as-yet-uncharacterized RNA-binding domain to the C-terminal region of that enzyme. Moreover, both U6-TUTase and RET 1 contain an $\mathrm{N}$-terminal C2H2-type zinc finger motif. In the case of RET 1, that zinc finger is essential for catalytic activity (Aphasizheva et al. 2004). In the case of the U6-TUTase, a potential role of the zinc finger motif for enzymatic activity remains to be elucidated. On the other hand, that zinc finger motif rather might supplement the adjacent RRM domain, in order to establish the observed unique substrate specificity of the human U6-TUTase. Furthermore, it is interesting to note that the TUTase "signature motif' (FGSS) (Aphasizheva et al. 2004), assigned to the catalytic core of RET 1, is also present within the human U6-TUTase (at position 208) (data not shown). In addition, adjacent to that element both enzymes share a triad of carboxylate (aspartate) residues, thought to represent the catalytical center of the RET 1 enzyme (Aphasizheva et al. 2004). Thus, it appears that in spite of their large phylogenetic distance and extended sequence divergence, these two eukaryotic TUTases still have preserved functionally important sequence elements.

With respect to the human U6-TUTase, another intriguing question is related to its biological function. A strictly controlled elongation reaction results in the restoration of the four $3^{\prime}$-terminal UMP-residues found in newly transcribed U6 snRNA (Trippe et al. 2003). Furthermore, U6 snRNA molecules from distant organisms, such as Drosophila and Caenorhabditis elegans but not yeast, are accepted as substrates by that human U6-TUTase (Trippe et al. 1998). Therefore, it was surprising that even a very extensive databank search did not reveal any indication for the existence of an ortholog in invertebrates (in vertebrates, only two close homologs could be identi- fied in mouse and rat genomes, with amino acid sequence identities of $77 \%$ and $74 \%$, respectively). Our findings may either indicate the absence of such a $3^{\prime}$-end modification of U6 snRNA in invertebrates or, alternatively, a highly divergent enzyme must be involved. This notion also holds true for the situation concerning the U6atac RNA of the minor spliceosome. That minor spliceosomal RNA is functionally equivalent to the abundant U6 snRNA. Since the U6atac RNA definitely is not accepted as substrate for the human U6-TUTase (Fig. 5), the life cycle of that minor RNA must show a mechanistic difference compared to that of the major U6 snRNA-possibly with respective consequences for the splicing process of U12 introns. Alternatively, a separate as-yet-unidentified TUTase may be responsible for the modification of U6atac RNA. As with the nondetectable U6-TUTase sequences in invertebrates, one would have to conclude again that such a putative U6atac RNA-specific TUTase in vertebrates should be highly divergent from the U6-TUTase described here, both by sequence and domain structure. In this context, the notion appears very intriguing that those rare U12 introns are absent from lower eukaryotes (Patel and Steitz 2003).

Finally, the results presented here indicate that the U6-TUTase constitutes an essential cellular protein. Apparently, this modifying enzyme accumulates within the nucleoli. At first glance, a predominant nucleolar localization of the overexpressed protein appeared quite unexpected. However, several reports have hypothesized that essential post-transcriptional modification steps of U6 snRNA biogenesis might occur within the nucleolus. These modifications involve 2'-O-methylation and pseudouridylation, directed by small nucleolar guide RNAs (Tycowski et al. 1998; Ganot et al. 1999). Furthermore, direct evidence for a transient nucleolar localization of U6 snRNA has been obtained (Lange and Gerbi 2000). Thus, in addition to biogenesis, our result of a predominant nucleolar localization of U6-TUTase may attribute a new recycling function to that nuclear subcompartment. On the other hand, U6 (and other) snRNPs and respective recycling factors have been found to accumulate within Cajal bodies (CB) (Bell et al. 2002; Stanek et al. 2003; Damianov et al. 2004). In this context, it is interesting to note the complete absence of U6-TUTase from those nuclear inclusions. It appears that individual steps of U6 snRNA biogenesis and recycling take place at different subnuclear compartments and involve extensive shuttling between nucleoli and nucleoplasm.

Further experiments, in particular the application to in vitro splicing assays of antibodies directed against U6-TUTase, should provide clues on the precise physiological significance of the U6-TUTase-catalyzed reaction. Such functional studies may also help to understand why that particular small RNA acquired its "own" modifying enzyme during evolution. 


\section{MATERIALS AND METHODS}

\section{Protein fractionation, TUTase assay, and RNA analysis}

Isolation of U6-TUTase from HeLa cell S100 extracts and purification by ion-exchange chromatography have been described in detail previously (Trippe et al. 2003). For affinity chromatography, oligo(A)/U6-3 RNA was coupled to oligo(dT)cellulose. To generate the corresponding template for in vitro transcription, the U6-3 coding sequence was amplified by PCR with an upstream primer containing an oligo $\mathrm{A}_{(20)}$-linker between the T7 promoter and the 5 '-end of the U6 snRNA sequence. Elution of the TUTase from the affinity column was with $10 \mu \mathrm{g} / \mathrm{mL}$ RNase A. The standard TUTase reaction $(50 \mu \mathrm{L})$ was performed in $60 \mathrm{mM} \mathrm{KCl}, 12 \mathrm{mM}$ HEPES/KOH ( $\mathrm{pH} 7.9$ ), $5 \mathrm{mM} \mathrm{MgCl}_{2}$, $2 \mathrm{mM}$ dithiothreitol, $0.1 \mathrm{mM}$ EDTA, 12\% (v/v) glycerol, and $5 \mu \mathrm{Ci}\left[\alpha-{ }^{32} \mathrm{P}\right] \mathrm{UTP}$, with a final concentration of $0.5 \mu \mathrm{M}$ UTP. Substrate RNAs were either $1 \mu \mathrm{g}$ of phenol-extracted total cellular RNA or 50 ng of U6-3 RNA, synthesized in vitro with T7 RNA polymerase (New England Biolabs). Synthesis of U6-3 RNA (referring to U6 snRNA molecules that contain three 3 '-terminal U-residues), analysis of purified RNA products in denaturing $6 \%$ polyacrylamide gels, and autoradiography were as outlined previously (Trippe et al. 1998). A cDNA clone of U6atac RNA (Schneider et al. 2002) was used to amplify the coding sequence by PCR, with two primers providing a $5^{\prime}$-flanking T7 promoter and a $3^{\prime}$-terminal DraI restriction site, respectively. After cloning into the pUC18 vector, restriction of the resulting plasmid with the DraI enzyme and transcription by T7 RNA polymerase produced U6atac RNA molecules containing three $3^{\prime}$-terminal UMP-residues.

\section{Sequencing of the purified protein}

After the final purification step in a 7.5\% SDS-PAGE (Trippe et al. 2003), the protein band was cut from the silver-stained gel. In-gel digestion of the protein with trypsin overnight was as described by Shevchenko et al. (1996). Prior to extraction, a peptide mass fingerprint of the main protein component was performed. For this, $0.5 \mu \mathrm{L}$ of the supernatant was analyzed by MALDI-ToF MS on a Bruker Reflex IV mass spectrometer using the thin layer technique on a polished stainless steel target for sample preparation (Shevchenko et al. 1996). Protein was identified by searching the measured peptide masses against the NCBInr database using Mascot as search engine. For extensive sequence analysis, the extracted peptides were analyzed in an LC-coupled ESI Q-ToF mass spectrometer (Q-ToF Ultima; Waters) under standard conditions. Proteins were identified by searching fragment spectra of sequenced peptides against the NCBInr database, again with Mascot.

\section{Analysis of Recombinant U6-TUTase}

The full-length cDNA coding for U6-TUTase was obtained from two cDNA clones provided by the German Resource Center (RZPD, Berlin) and inserted in frame into the T-Rex expression vector ( $\mathrm{pcDNA} / \mathrm{TO} / \mathrm{myc}-\mathrm{His}$; Invitrogen) providing C-terminal myc- and His-tags. Transfection of HeLa cells was by calcium phosphate/DNA coprecipitation, and cells were harvested after $48 \mathrm{~h}$. Recombinant U6-TUTase was purified from S100 extracts of transfected cells under nondenaturing conditions, using the nickel nitrilotriacetic acid (Ni-NTA) affinity purification system (QIAGEN). Step-elution of proteins was with imidazole. Prior to TUTase assays, fractions were dialyzed against buffer D $(20 \mathrm{mM}$ HEPES/KOH at $\mathrm{pH} 7.9,100 \mathrm{mM} \mathrm{KCl}, 0.2 \mathrm{mM}$ EDTA, $0.5 \mathrm{mM}$ DTT, and $20 \%$ [v/v] glycerol). For Western blots, proteins were fractionated in $7.5 \%$ SDS-PAGE, transferred to nitrocellulose membranes (Schleicher \& Schuell), and immunostained with mouse anti-myc 9E10 antibody (1:5000 dilution) and anti-mouse antibody-peroxidase (1:5000 dilution; Sigma). Signals were detected by enhanced chemiluminescence (Amersham Pharmacia Biotech). Custom-made polyclonal rabbit antisera against U6-TUTase were raised and purified by Eurogentec (Belgium).

\section{siRNA and cell proliferation}

siRNA duplexes either targeting firefly (Photonis pyralis) luciferase (GL2) or human spliceosomal factor Prp8 (hPrp8) mRNA were designed and synthesized in house using $5^{\prime}$-silyl, 2'-ACE phosphoramidites (Dharmacon) and annealed as described previously (Elbashir et al. 2002; Yuan et al. 2004). siRNA directed against U6-TUTase mRNA was designed and synthesized by QIAGEN. A BLAST search against the human genome (NCBI UniGene database) identified the gene of interest as the only target. The following 21-nt siRNA duplexes were used (only sense strand shown):

- U6-TUTase (NM_022830): 5'-GCAGCCAAUUACUGCCGAAdTdT-3'

- hPrp8 (NM_006445): 5'-GCCCAUCAACGGAGCCAUCdTdT-3'

Using Oligofectamine (Invitrogen), transient transfections of siRNAs into HeLa SS6 cells (Elbashir et al. 2002) were performed at different cell confluencies: 20,000 cells/well in 96-well plates for cell proliferation or 300,000 cells/well in six-well plates for realtime RT-PCR analysis after $48 \mathrm{~h}$. The effect of depletion of U6-TUTase and of hPrp8 on cell proliferation was monitored by determining the number of viable cells of the knockdown in comparison to control cells treated with siRNA against GL2. After $24 \mathrm{~h}, 48 \mathrm{~h}$, and $72 \mathrm{~h}$, the number of viable cells was determined by using a cell counter (CASYcounterTT; Schärfe Systems GmbH). The rates were depicted relative to the number of viable control (GL2) cells at $72 \mathrm{~h}$. To determine knockdown efficiencies, cells were assayed at $48 \mathrm{~h}$ after transfection. U6-TUTase knockdown was determined by quantification of relative amounts of target mRNA in knockdown cells versus target mRNA in control (GL2) cells with one-step real-time RT-PCR using SYBR green. For normalization, the intronless glutamate dehydrogenase 2 (GLUD2) gene was used as internal reference. Total RNA was isolated from HeLa SS6 cells transfected either with control (GL2) or U6-TUTase siRNAs (RNeasy Mini Kit; QIAGEN) and in-column treated with DNase I (QIAGEN). The following PCR primers were used:

- U6-TUTase (f): 5' -GGACAGAAAAACTGCTGTGAGG-3'

- U6-TUTase (r): 5'-TGCACCATCTCTCCAACCTCTA-3'

- GLUD2 (f): 5' -TCGTGGAGGACAAGTTGGTG-3'

- GLUD2 (r): 5'-TTGCAGGGCTTGATGATCCG-3'

Real-time PCRs were performed in a DNA Engine Option 1 System (Bio-Rad Laboratories). Specificity of products was 
verified by high-resolution gel electrophoresis and resulted in a single product of expected length. In addition, melting curves analyzed in parallel showed single product-specific melting curves. Transcripts revealed real-time PCR efficiency rates for U6-TUTase (2.03) and GLUD2 (2.06) in the investigated range from 0.2 to $25 \mathrm{ng}$ of total RNA input $(n=2)$, with high linearity (Pearson correlation coefficient $r>0.99$ ). Relative quantification of knockdown versus control mRNA levels was by the mathematical model described by Pfaffl (2001). Additional controls without RT-mix excluded residual DNA contamination.

\section{Immunofluorescence and confocal laser scanning microscopy}

Cell culture, fluorescence microscopy, indirect immunofluorescence, and visualization of samples were performed as outlined previously (Andrei et al. 2005). The following primary antibodies were used: rabbit anti-NOP58 (1:600 dilution) (Watkins et al. 2000); mouse monoclonal anti-c-myc (9E10, 1:400; Santa Cruz Biotechnlogy); and rabbit anti-p110 (1:500) (Schaffert et al. 2004). Secondary antibodies were: Alexa-488 goat anti-mouse and Texas Red chicken anti-rabbit IgG $(\mathrm{H}+\mathrm{L})$ (1:500) conjugates (Molecular Probes).

\section{ACKNOWLEDGMENTS}

This work was supported by a grant from the Deutsche Forschungsgemeinschaft (Be 531/19-2).

Received March 14, 2006; accepted May 4, 2006.

\section{REFERENCES}

Andrei, M.A., Ingelfinger, D., Heintzmann, R., Achsel, T., RiveraPomar, R., and Lührmann, R. 2005. A role for eIF4E and eIF4Etransporter in targeting mRNPs to mammalian processing bodies. RNA 11: 717-722.

Andrews, N.C. and Baltimore, D. 1986. Purification of a terminal uridylyltransferase that acts as host factor in the in vitro poliovirus replicase reaction. Proc. Natl. Acad. Sci. 83: 221-225.

Aphasizhev, R., Sbicego, S., Peris, M., Jang, S.H., Aphasizheva, I., Simpson, A.M., Rivlin, A., and Simpson, L. 2002. Trypanosome mitochondrial $3^{\prime}$ terminal uridylyl transferase (TUTase): The key enzyme in U-insertion/deletion RNA editing. Cell 108: 637-648.

Aphasizhev, A., Aphasizheva, I., Nelson, R.E., Gao, G., Simpson, A.M., Kang, X., Falick, A.M., Sbicego, S., and Simpson, L. 2003. Isolation of a U-insertion/deletion editing complex from Leishmania tarentolae mitochondria. EMBO J. 22: 913-924.

Aphasizheva, I., Aphasizhev, R., and Simpson, L. 2004. RNA editing terminal uridylyl transferase 1: Identification of functional domains by mutational analysis. J. Biol. Chem. 279: 24123-24130.

Aravind, L. and Koonin, E.V. 1999. DNA polymerase $\beta$-like nucleotidyltransferase superfamily: Identification of three new families, classification and evolutionary history. Nucleic Acids Res. 27: 1609-1618.

Bell, M., Schreiner, S., Damianov, A., Reddy, R., and Bindereif, A. 2002. p110, a novel human U6 snRNP protein and U4/U6 snRNP recycling factor. EMBO J. 21: 2724-2735.

Booth, B.L. and Pugh, B.F. 1997. Identification and characterization of a nuclease specific for the $3^{\prime}$ end of the U6 small nuclear RNA. J. Biol. Chem. 272: 984-991.

Brow, D.A. 2002. Allosteric cascade of spliceosome activation. Annu. Rev. Genet. 36: 333-360.
Brow, D.A. and Guthrie, C. 1988. Spliceosomal RNA U6 is remarkably conserved from yeast to mammals. Nature 334: 213-218.

Dahlberg, J.E. and Lund, E. 1988. The genes and transcription of the major small nuclear RNAs. In Structure and function of the major and minor small nuclear ribonucleoprotein particles (ed. M.L. Birnstiel), pp. 38-70. Springer-Verlag, Berlin.

Damianov, A., Schreiner, S., and Bindereif, A. 2004. Recycling of the U12-type spliceosome requires p110, a component of the U6atac snRNP. Mol. Cell. Biol. 24: 1700-1708.

Elbashir, S.M., Harborth, J., Weber, K., and Tuschl, T. 2002. Analysis of gene function in somatic mammalian cells using small interfering RNAs. Methods 26: 199-213.

Ernst, N.L., Panicucci, B., Igo Jr., R.P., Panigrahi, A.K., Salavati, R., and Stuart, K. 2003. TbMP57 is a $3^{\prime}$ terminal uridylyl transferase (TUTase) of the Trypanosoma brucei editosome. Mol. Cell 11: $1525-1536$.

Ganot, P., Jady, B.E., Bortolin, M.-L., Darzacq, X., and Kiss, T. 1999. Nucleolar factors direct the 2'-O-ribose methylation and pseudouridylation of U6 spliceosomal RNA. Mol. Cell. Biol. 19: 69066917.

Grainger, R.J. and Beggs, J.D. 2005. Prp8 protein: At the heart of the spliceosome. RNA 11: 533-557.

Hartmuth, K., Urlaub, H., Vornlocher, H.P., Will, C.L., Gentzel, M., Wilm, M., and Lührmann, R. 2002. Protein composition of human prespliceosomes isolated by a tobramycin affinity-selection method. Proc. Natl. Acad. Sci. 99: 16719-16724.

Jurica, M.S., Licklider, L.J., Gygi, S.R., Grigorieff, N., and Moore, M.J. 2002. Purification and characterization of native spliceosomes suitable for three-dimensional structural analysis. RNA 8: 426439.

Kunkel, G.R., Maser, R.L., Clavet, J.P., and Pederson, T. 1986. U6 small nuclear RNA is transcribed by RNA polymerase III. Proc. Natl. Acad. Sci. 83: 8575-8579.

Lange, T.S. and Gerbi, S.A. 2000. Transient nucleolar localization of U6 small nuclear RNA in Xenopus laevis oocytes. Mol. Biol. Cell 11: 2419-2428.

Lund, E. and Dahlberg, J.E. 1992. Cyclic 2'-3'-phosphates and nontemplated nucleotides at the $3^{\prime}$ end of spliceosomal U6 small nuclear RNAs. Science 255: 327-330.

Martin, G. and Keller, W. 1996. Mutational analysis of mammalian poly(A) polymerase identifies a region for primer binding and catalytic domain, homologous to the family $\mathrm{X}$ polymerases and to other nucleotidyltransferases. EMBO J. 15: 2593-2603.

Panigrahi, A.K., Schnaufer, A., Ernst, N.L., Wang, B., Carmean, N., Salavati, R., and Stuart, K. 2003. Identification of novel components of Trypanosoma brucei editosomes. RNA 9: 484-492.

Patel, A.A. and Steitz, J.A. 2003. Splicing double: Insights from the second spliceosome. Nat. Rev. Mol. Cell Biol. 4: 960-970.

Perumal, K., Sinha, K., Henning, D., and Reddy, R. 2001. Purification, characterization and cloning of the cDNA of human srp RNA 3'-adenylating enzyme. J. Biol. Chem. 276: 21791-21796.

Pfaffl, M.W. 2001. A new mathematical model for relative quantification in real-time RT-PCR. Nucleic Acids Res. 29: e45.

Reddy, R., Henning, D., Das, G., Harless, M., and Wright, D. 1987. The capped U6 small nuclear RNA is transcribed by RNA polymerase III. J. Biol. Chem. 262: 75-81.

Rinke, J. and Steitz, J.A. 1985. Association of the lupus antigen La with a subset of U6 snRNA molecules. Nucleic Acids Res. 13: 2617-2629.

Schaffert, N., Hossbach, M., Heintzmann, R., Achsel, T., and Lührmann, R. 2004. RNAi knockdown of hPrp31 leads to an accumulation of U4/U6 di-snRNPs in Cajal bodies. EMBO J. 23: 3000-3009.

Schneider, C., Will, C.L., Kakarova, O.V., Makarov, E.M., and Lührmann, R. 2002. Human U4/U6.U5 and U4atac/U6atac.U5 tri-snRNPs exhibit similar protein compositions. Mol. Cell. Biol. 22: 3219-3229.

Shevchenko, A., Wilm, M., Vorm, O., and Mann, M. 1996. Mass spectrometric sequencing of proteins from silver-stained polyacrylamide gels. Anal. Chem. 68: 850-858. 
Staley, J.P. and Guthrie, C. 1998. Mechanical devices of the spliceosome: Motors, clocks, springs and things. Cell 92: 315-326.

Stanek, D., Rader, S.D., Klingauf, M., and Neugebauer, K.M. 2003. Targeting of U4/U6 small nuclear RNP assembly factor SART3/p110 to Cajal bodies. J. Cell Biol. 160: 505-516.

Tazi, J., Forne, T., Janteur, P., Cathala, G., and Brunel, C. 1993. Mammalian U6 small nuclear RNA undergoes $3^{\prime}$ end modifications within the spliceosome. Mol. Cell. Biol. 13: 16411650.

Trippe, R., Sandrock, B., and Benecke, B.-J. 1998. A highly specific terminal uridylyl transferase modifies the 3 '-end of U6 small nuclear RNA. Nucleic Acids Res. 26: 3119-3126.

Trippe, R., Richly, H., and Benecke, B.-J. 2003. Biochemical characterization of a U6 small nuclear RNA-specific terminal uridylyl transferase. Eur. J. Biochem. 270: 971-980.

Tycowski, R.T., You, Z.-H., Graham, P.J., and Steitz, J.A. 1998. Modification of U6 spliceosomal RNA is guided by other small RNAs. Mol. Cell 2: 629-638.
Watkins, N.J., Ségault, V., Charpentier, B., Nottrott, S., Fabrizio, P., Bachi, A., Wilm, M., Rosbash, M., Branlant, C., and Lührmann, R. 2000. A common core RNA structure shared between the small nucleolar box C/D RNPs and the spliceosomal U4 snRNP. Cell 103: 457-466.

Yuan, B., Latek, R., Hossbach, M., Tuschl, T., and Lewitter, F. 2004. SiRNA selection server: An automated siRNA oligonucleotide prediction server. Nucleic Acids Res. 32: w130-w134.

Zabel, P., Dorssers, L., Wernars, K., and VanKammen, A. 1981. Terminal uridylyl transferase of. Vigna unguiculata Purification and characterization of an enzyme catalyzing the addition of a single UMP residue to the $3^{\prime}$-end of an RNA primer. Nucleic Acids Res.. 9: 2433-2453.

Zhelkovsky, A.M., Kessler, M.M., and Moore, C.L. 1995. Structurefunction relationships in the Saccharomyces cerevisiae poly(A) polymerase. Identification of a novel RNA binding site and a domain that interacts with specificity factor(s). J. Biol. Chem. 270: 26715-26720. 

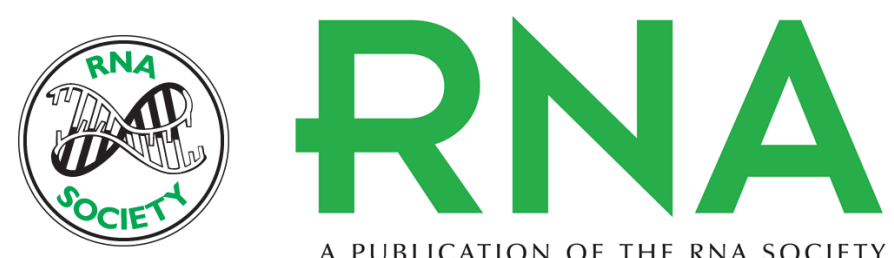

A PUBLICATION OF THE RNA SOCIETY

\section{Identification, cloning, and functional analysis of the human U6 snRNA-specific terminal uridylyl transferase}

Ralf Trippe, Elena Guschina, Markus Hossbach, et al.

RNA 2006 12: 1494-1504

References This article cites 39 articles, 21 of which can be accessed free at:

http://rnajournal.cshlp.org/content/12/8/1494.full.html\#ref-list-1

License

Email Alerting Receive free email alerts when new articles cite this article - sign up in the box at the Service top right corner of the article or click here.

To subscribe to RNA go to:

http://rnajournal.cshlp.org/subscriptions 